\title{
GENETIC POLYMORPHISM OF HUMAN PLASMINOGEN IN JAPANESE: CORRESPONDENCE OF ALLELES THUS FAR REPORTED IN JAPANESE AND DIFFERENCE OF ACTIVITY AMONG PHENOTYPES
}

\author{
Toshinori Nishigaki and Keiichi Omoto \\ Department of Anthropology, Faculty of Science, \\ The University of Tokyo, Tokyo 113, Japan
}

\begin{abstract}
Summary Genetic polymorphism of human plasminogen in Japanese was investigated in 373 plasma samples from blood donors using polyacrylamide gel isoelectric focusing followed by immunofixation. It was confirmed that two alleles which had not been described in Caucasoid and Negroid populations exist in Japanese. The correspondence of the alleles thus far described also was examined by comparison runs. As a result, it was revealed that $P L G^{2 \prime}$ described by Nakamura et al. (1980a) is identical with $P L G^{2}$ described by Nishimukai et al. (1981) and that $P L G^{C}$ reported as a rare variant by Nishimukai et al. (1982) corresponds to $P L G^{2}$ described by Hobart (1979). Allele frequencies were estimated as follows: $P L G N^{*} 1=$ $0.958, P L G N^{*} 2=0.004, P L G N^{*} 3=0.023, P L G N^{*} 4=0.015$.

Caseinolytic activities per milligram antigen protein of different phenotypes were measured, and that of type 1-3 was about half of others. Therefore, the products of $P L G N * 3$ seem to have no or much lower activity than those of other alleles.
\end{abstract}

\section{INTRODUCTION}

Plasminogen is a zymogen in plasma which is converted into proteolytic enzyme, plasmin by plasminogen activators. It is known that the main function of plasmin is the degradation of fibrinogen and the fibrin blood clot.

Genetic polymorphism of plasminogen was first investigated in English and Gambians by Hobart (1979) using isoelectric focusing followed by zymogram technique, and two alleles $P L G^{I}$ and $P L G^{2}$ were described. Raum et al. $(1979,1980)$ investigated Black, White and Oriental in U.S.A. and also reported two alleles, $P L G N^{*} A$ and $P L G N^{*} B$, and some rare alleles.

Genetic polymorphism of plasminogen in Japanese population was recently reported by Nakamura et al. (1980a, b), Nishimukai et al. (1981) and Nakamura and

Received May 17, 1982 
Abe (1982). Besides two alleles reported in Caucasoid and Negroid populations, Nakamura et al. (1980b) described two alleles $P L G^{\prime \prime}$ and $P L G^{2}$, and Nishimukai et al. (1981) described an allele $P L G^{B}$. Subsequently, Nishimukai et al. (1982) reported three variants $P L G^{3}, P L G^{M}$ and $P L G^{C}$ in Japanese. However, two groups reported their study independently and adopted different nomenclature. In the present study, we investigated the polymorphism of plasminogen in Japanese and examined the correspondence of the alleles thus far reported.

Nakamura et al. (1980b) and Nishimukai et al. (1982) described that one of the phenotypes observed in Japanese is not distinguishable from PLG 1 by caseinolytic patterns, and Nakamura and Abe (1982) measured plasminogen activity by a fibrinolytic radial diffusion technique using fibrin plate and reported that the products of $P L G^{\prime \prime}$ have no or less plasminogen activity. Caseinolytic activities per milligram antigen protein of different phenotypes were also measured in the present study.

\section{MATERIALS AND METHODS}

Samples. A total of 373 plasma samples from unrelated blood donors were used for population study. Samples were obtained in $\mathrm{ACD}$, and stored at $-30^{\circ} \mathrm{C}$ until use.

Samples of $15 \mu \mathrm{l}$ were treated with $5 \mu \mathrm{l}$ neuraminidase at $1 \mathrm{U} / \mathrm{ml}$ (Sigma, type $\mathrm{V}$, St. Louis, Mo.) in phosphate-buffered saline at $\mathrm{pH} 6.8$ for $10 \mathrm{hr}$ at $37^{\circ} \mathrm{C}$.

Isoelectric focusing electrophoresis. 2.2\% Ampholine (LKB) (6 parts $\mathrm{pH} 6-8$, 1 part $\mathrm{pH} 3.5-10$ ) was incorporated into $5.0 \%$ polyacrylamide gel (thickness $1 \mathrm{~mm}$ ) with $0.2 \mathrm{M}$ taurine (Hobart et al., 1975). The gel was photopolymerized with riboflavin. After prefocusing at $500 \mathrm{~V}$ constant voltage for $1 \mathrm{hr}$ (electrode distance $9 \mathrm{~cm}$ ), ca. $7 \mu$ of plasma samples were applied with pieces of filter paper at anodal side of the gel, and the voltage was raised to $650 \mathrm{~V}$. After $1 \mathrm{hr}$, filter paper pieces were removed and the voltage was raised and kept constant at $900 \mathrm{~V}$ for $3 \mathrm{hr}$.

Immunofixation. After electrophoresis, plasminogen was localized by immunofixation with a $50 \%(\mathrm{v} / \mathrm{v})$ solution of rabbit anti-human plasminogen antiserum (Atlantic Antibodies, Scarborough, Me.), essentially following the method described by Raum et al. (1980).

Assay for plasminogen activity. Plasma plasminogen was assayed by the caseinolytic method (McNicol and Douglas, 1976). $0.5 \mathrm{ml}$ of the test plasma was mixed and incubated with $0.5 \mathrm{ml}$ of $1 / 6 \mathrm{~N} \mathrm{HCl}$ for 15 min at room temperature and neutralized with $0.5 \mathrm{ml} 1 / 6 \mathrm{~N} \mathrm{NaOH}$ and $1 \mathrm{ml}$ of phosphate-buffered saline $(0.1 \mathrm{M}$ phosphate, $0.9 \% \mathrm{NaCl}$, pH 7.6). Then, $0.5 \mathrm{ml}$ of 2,000 U/ml streptokinase (Sigma, St. Louis, Mo.) and $2 \mathrm{ml}$ of $5 \%$ casein (acc. Hammarsten, Wako Pure Chemical, Osaka, Japan) were added. The mixture of acid-treated plasma with streptokinase and casein was incubated at $37^{\circ} \mathrm{C}$. After $2 \min 2 \mathrm{ml}$ was withdrawn and mixed with $3 \mathrm{ml}$ of $15 \%$ trichloroacetic acid. This mixture served as the control. After $62 \mathrm{~min}$ incubation of the original mixture a further $2 \mathrm{ml}$ sample was added to $3 \mathrm{ml}$ of $15 \%$ 
trichloroacetic acid. Each mixture was allowed to stand for $30 \mathrm{~min}$ at room temperature and then the precipitates were removed by centrifugation, and the optical density of each solution was measured at $280 \mathrm{~nm}$. The results were expressed in casein unit defined as the amount of enzyme which liberated $450 \mu \mathrm{g}$ of trichloroacetic acid-soluble tyrosine in $1 \mathrm{hr}$ under the condition of assay (Remmert and Cohen, 1949; Robbins and Summaria, 1976).

Assay for plasminogen concentration. Plasminogen concentration was measured by the single radial immunodiffusion technique of Mancini et al. (1965). Five microliters of plasma sample was placed in a hole $(2 \mathrm{~mm}$ diameter $)$ in the $1.8 \mathrm{mn}$ thick layer of $1.5 \%(\mathrm{w} / \mathrm{v})$ agarose gel made with $0.05 \mathrm{M}$ barbital buffer ( $\mathrm{pH} 8.6$, containing $0.05 \%$ sodium azide) containing $0.5 \%$ anti-plasminogen antiserum. After incubation at room temperature for $72 \mathrm{hr}$, diameters of the immunoprecipitate rings were measured. Protein Standard Plasma (Behringwerke, Marburg, W. Germany) was used for calibration.

\section{RESULTS AND DISCUSSION}

Six different immunofixation patterns were observed by the present survey of 373 plasma samples from blood donors living in Tokyo (Fig. 1). The samples which produced immunofixation patterns in Fig. 1 were subjected to neuraminidase treatment and examined by isoelectric focusing electrophoresis (pH 3.5-10) (Fig. 2). The number of bands of each phenotype reduced by neuraminidase treatment, however, each neuraminidase-treated sample contained genetic variation which was not attributable to postsynthetic sialidation (Fig. 2). In order to identify the phenotypes with those reported by Hobart (1979), plasma samples from Caucasoid individuals living in Tokyo were used in comparison runs. As a result, two of six phenotypes

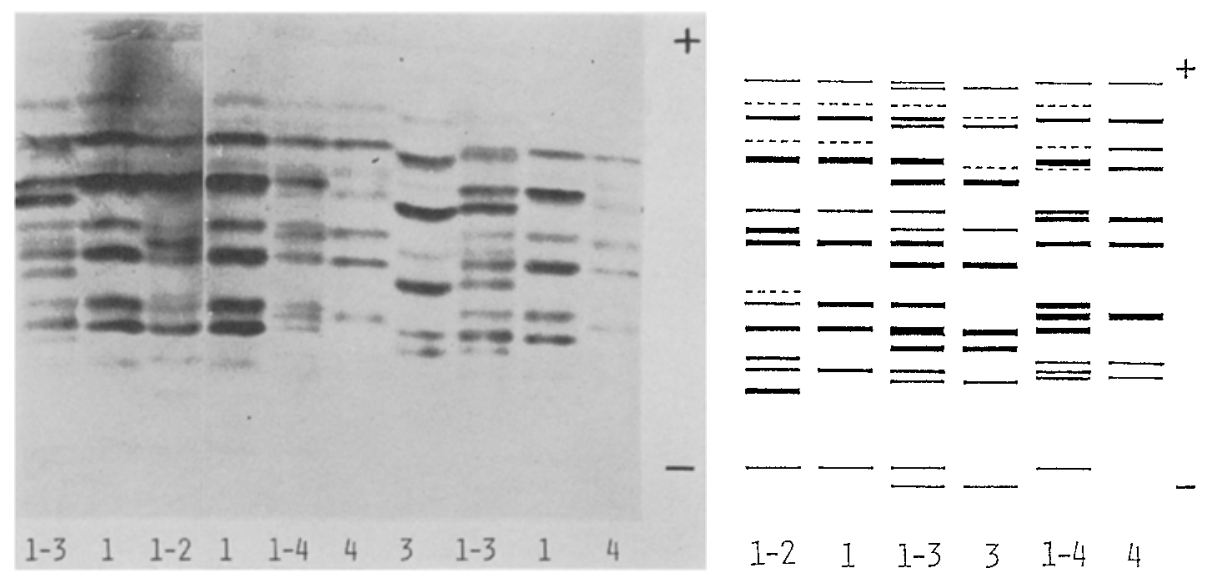

Fig. 1. Photograph and diagram showing plasminogen phenotypes obtained by isoelectric focusing and subsequent immunofixation. 
(A)

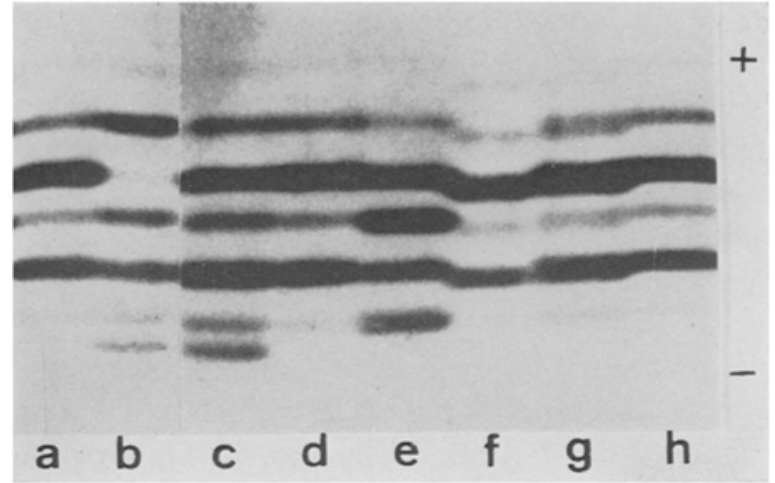

(B)

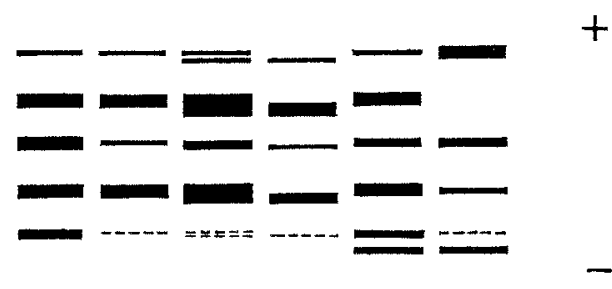

$$
\begin{array}{llllll}
1-2 & 1 & 1-3 & 3 & 1-4 & 4
\end{array}
$$

Fig. 2. Photograph (A) and diagram (B) showing immunofixation patterns of neuraminidase treated plasma samples. (A) a, d and h, PLGN 1; b, PLGN 4; c, PLGN 1-4; e, PLGN 1-2; f, PLGN 3; g, PLGN 1-3.

were identical with type 1 and 2-1 reported by Hobart (1979). Judging from each pattern of untreated and neuraminidase-treated samples (Figs. 1 and 2), other four phenotypes were considered to be two homozygotes of two kinds of allele and two heterozygotes of $P L G N^{*} 1$ and these two alleles. We referred to two new alleles as $P L G N^{*} 3$ and $P L G N^{*} 4$ on the basis of nomenclature proposed by Hobart (1979). According to the patterns of untreated and treated samples (Figs. 1 and 2), $P L G N^{*} 3$ seems to be more basic variant than $P L G N^{*} 1$, while $P L G N^{*} 4$ seems to be more acidic variant than $P L G N^{*} l$.

Plasma samples typed 1-1', 2-1 and 2'-1 by Dr. Nakamura and those typed 1-B, 2-1 and 1-C by Dr. Nishimukai were also used in comparison runs to confirm the correspondence of four alleles observed in the present study to those thus far reported in Japanese population (Nakamura et al., 1980a, b; Nishimukai et al., 1981; Nakamura and Abe, 1982; Nishimukai et al., 1982). As a result of comparison runs, it is showed that plasma samples which Nishimukai et al. (1981) had described to be identical with PLG 2-1 reported by Hobart (1979) were not identical with it and that type 1-C reported by Nishimukai et al. (1982) was actually identical with it. Type 2-1 reported by Nishimukai (1981) and type 2'-1 reported by Nakamura et al. $(1980 \mathrm{a}, \mathrm{b})$ and Nakamura and Abe (1982) were identical with type 1-4 in this paper, 
and type 2-1 reported by Nakamura et al. (1980a, b) and Nakamura and Abe (1982) was identical with PLG 2-1 reported by Hobart (1979). On the basis of these results, the correspondence of four alleles in this paper to those thus far reported is presented in Table 1. It is not clear whether any of the rare acidic and rare basic variants reported by Raum et al. (1980) corresponds to either $P L G N^{*} 3$ or $P L G N^{*} 4$, since the photograph of their patterns was not presented by Raum et al. (1980).

The nomenclature proposed by Nishimukai et al. (1981) and Nishimukai et al. (1982) was not correct as described above, and in the nomenclature proposed by Nakamura et al. $(1980 \mathrm{a}, \mathrm{b})$ the relationship between $P L G^{1}$ and $P L G^{\prime \prime}$ and that between $P L G^{2}$ and $P L G^{2 \prime}$ were uncertain. Therefore, we proposed another nomenclature described above on the basis of Hobart's nomenclature (1979).

The results of the population study are presented in Table 2. A homozygous

Table 1. Correspondence of four alleles in this report to those thus far described.

\begin{tabular}{lcccc}
\hline & $P L G N^{*} I$ & $P L G N^{*} 2$ & $P L G N^{*} 3$ & $P L G N^{*} 4$ \\
\hline Hobart (1979) & $P L G^{\prime \prime}$ & $P L G^{2 \prime}$ & & \\
Raum et al. (1980) & $P L G N^{*} A$ & $P L G N^{*} B$ & & \\
Nakamura et al. (1980a, b) & $P L G^{1}$ & $P L G^{2}$ & $P L G^{\prime \prime}$ & $P L G^{2 \prime}$ \\
Nakamura and Abe (1982) & & & & \\
Nishimukai et al. (1981) & $P L G^{1}$ & & $P L G^{\mathrm{B}}$ & $P L G^{2}$ \\
Nishimukai et al. (1982) & & $P L G \mathrm{C}$ & & \\
\hline
\end{tabular}

Table 2. Distribution of plasminogen phenotypes and allele frequencies in a Japanese population.

\begin{tabular}{crcr}
\hline Phenotype & Obs. No. & $\%$ & Exp. No. \\
\hline 1 & 344 & 92.2 & 342.65 \\
$1-2$ & 3 & 0.8 & 2.88 \\
$1-3$ & 15 & 4.0 & 16.29 \\
$1-4$ & 9 & 2.4 & 10.54 \\
2 & 0 & 0 & 0.01 \\
$2-3$ & 0 & 0 & 0.07 \\
$2-4$ & 0 & 0 & 0.04 \\
3 & 1 & 0.3 & 0.19 \\
$3-4$ & 0 & 0 & 0.25 \\
4 & 1 & 0.3 & 0.08 \\
\hline Total & 373 & 100.0 & 373.00 \\
\hline
\end{tabular}

Allele frequencies: $\quad P L G N^{*} 1=0.958$

$P L G N^{*} 2=0.004$

$P L G N^{*} 3=0.023$

$P L G N^{*} 4=0.015$

Vol. 27, No. 4, 1982 
Table 3. Allele frequencies of plasminogen in various populations.

\begin{tabular}{lrllcccl}
\hline Population & $\mathrm{N}$ & $P L G N^{*} I$ & $P L G N^{*} 2$ & $P L G N^{*} 3$ & $P L G N^{*} 4$ & $P L G N^{*} R$ & Authors \\
\hline English & 327 & 0.71 & 0.29 & - & - & - & Hobart (1979) \\
Gambians & 89 & 0.86 & 0.14 & - & - & - & \\
Black & 127 & 0.795 & 0.193 & & & $0.012^{\text {a }}$ & Raum et al. (1980) \\
White & 102 & 0.686 & 0.299 & & & $0.015^{\mathrm{a}}$ & \\
Oriental & 69 & 0.962 & 0.029 & & & $0.007^{\mathrm{a}}$ & \\
Japanese & 258 & 0.958 & - & 0.022 & 0.020 & - & Nishimukai et al. (1981) \\
Japanese & 750 & 0.9560 & 0.0113 & 0.0233 & 0.0094 & - & Nakamura and Abe (1982) \\
Japanese & 373 & 0.958 & 0.004 & 0.023 & 0.015 & - & present study \\
\hline
\end{tabular}

a All rare alleles were grouped together as $P L G N^{*} R$.

Table 4. Plasminogen activity in individuals of known phenotypes.

\begin{tabular}{cccc}
\hline \multirow{2}{*}{ Phenotype } & No. & \multicolumn{2}{c}{ Specific activity $(\mathrm{U} / \mathrm{mg})^{\mathrm{a}}$} \\
\cline { 3 - 4 } & & Mean $\pm \mathrm{SE}$ & Range \\
\hline 1 & 16 & $15.1 \pm 2.4$ & $12.2 \pm 19.2$ \\
$1-2$ & 2 & 14.6 & $14.2,15.0$ \\
$1-3$ & 4 & $6.5 \pm 0.8$ & $5.6-7.4$ \\
$1-4$ & 1 & 15.8 & \\
\hline
\end{tabular}

a Caseinolytic activity per milligram antigen protein.

type 3 and homozygous type 4 were observed (Fig. 1). The allele frequencies of PLGN*1, PLGN*2, PLGN*3 and PLGN*4 were estimated to be $0.958,0.004,0.023$ and 0.015 , respectively. According to Table 1, the allele frequencies of plasminogen in various populations were compared (Table 3 ). In comparison with Caucasoid or Negroid population, the allele frequency of $P L G N^{*} 1$ is high and that of $P L G N^{*} 2$ is much lower in Japanese, while $P L G N^{*} 3$ and $P L G N^{*} 4$ which have not been observed in other populations exist in Japanese at a high level. It is presumably due to the low frequency of $P L G N^{*} 2$ in Japanese that Nishimukai et al. (1981) did not observe $P L G N^{*} 2$. The frequency of $P L G N^{*} 2$ in the present study is seen to be lower than that reported by Nakamura and Abe (1982), however, the difference between two reports is not statistically significant $\left(\chi^{2}=3.01\right.$, d.f. $\left.=1,0.07<p<0.1\right)$.

It is thus far described that the product of the allele which corresponds to $P L G N^{*} 3$ in this paper have no or less activity. In the present study, caseinolytic activity per milligram antigen protein was measured, and the results are presented in Table 4. The means of specific activity of each phenotype were as follows: PLGN 1, $15.1 \mathrm{U} / \mathrm{mg}$; PLGN 1-2, $14.6 \mathrm{U} / \mathrm{mg}$; PLGN 1-3, 6.5 U/mg; PLGN 1-4, $15.8 \mathrm{U} / \mathrm{mg}$. Four heterozygotes, PLGN1-3, of which activities were measured in the present study 
had the same level of plasminogen antigen concentration as other types, and no compensatory increase of plasminogen was observed. These results that caseinolytic activity per milligram antigen protein of type 1-3 was about half of others (Table 4) are quite similar to those reported by Nakamura and Abe (1982), and although the activity of type 3 was not measured in the present study, the products of $P L G N^{*} 3$ seem to have no or much lower activity than those of others.

The allele frequency of $P L G N^{*} 3$ in Japanese is polymorphic, and a homozygote of $P L G N^{*} 3$ was observed in the present population study of plasma samples from blood donors. Abnormal plasminogen with low activity was previously found in Japanese patients with recurrent thrombosis (Aoki et al., 1978, Sakata and Aoki, 1980; Kazama et al., 1981), however, it is not known whether the products of $P L G N^{*} 3$ are identical with abnormal plasminogen thus far reported. Further investigations on the relation between the phenotypes with lower activity and diseases seem to be necessary, and the surveys of genetic polymorphism of plasminogen in other populations are also necessary to solve the question whether the distribution of $P L G N^{*} 3$ is only in Japanese population or expands into all Mongoloid populations.

Acknowledgment We wish to express our thanks to Mr. M. Uchikawa, Blood Transfusion Service, Tokyo University Hospital, for providing us with blood samples. We are also indebted to Prof. K. Abe and Dr. S. Nakamura, Department of Legal Medicine, Tokyo Women's Medical College and Dr. H. Nishimukai, Department of Legal Medicine, Kyoto Prefectural University of Medicine, for providing us with the plasma samples of different phenotypes for comparison runs.

\section{REFERENCES}

Aoki, N., Moroi, M., Sakata, Y., and Yoshida, N. 1978. Abnormal plasminogen. A hereditary molecular abnormality found in a patient with recurrent thrombosis. J. Clin. Invest. 61: 11861195.

Hobart, M.J., Lachmann, P.J., and Alper, C.A. 1975. Polymorphism of human C6. in Protides of the Biological Fluids. Peeters, H., ed., Pergamon Press, New York, pp. 575-580.

Hobart, M.J. 1979. Genetic polymorphism of human plasminogen. Ann. Hum. Genet., Lond. 42: 419-423.

Kazama, M., Tahara, C., Suzuki, Z., Gohchi, K., and Abe, T. 1981. Abnormal plasminogen, a case of recurrent thrombosis. Thrombosis Res. $21: 517-522$.

Mancini, G., Carbonara, A.O., and Heremans, F. 1965. Immunochemical quantitation of antigens by single radial immunodiffusion. Int. J. Immunochem. 2: 235-254.

McNicol, G.P. and Douglas, A.S. 1976. Plasminogen assay in plasma. in Human Blood Coagulation, Haemostasis and Thrombosis. 2nd ed., Biggs, R., ed., Blackwell Scientific Publication, Ltd., Oxford, pp. 724-726.

Nakamura, S., Abe, K., Ooue, O., Akiyama, K., Yamadate, N., and Yamashita, K. 1980a. Genetic polymorphism of human plasminogen in Japanese. J. Tokyo Wom. Med. Coll. 50: 276-279 (in Japanese).

Nakamura, S., Abe, K., Ooue, O., Akiyama, K., Yamadate, N., and Yamashita, K. 1980b. Genetic polymorphism of human plasminogen in Japanese (II). Study of plasminogen polymorphism using immunofixation electrophoresis. J. Tokyo Wom. Med. Coll. 50: 450-454 (in Japanese).

Nakamura, S. and Abe, K. 1982. Genetic polymorphism of human plasminogen in the Japanese 
population: New plasminogen variants and relationship between plasminogen phenotypes and their biological activities. Hum. Genet. 60: 57-59.

Nishimukai, H., Kera, Y., Sakata, K., and Yamasawa, K. 1981. Genetic polymorphism of plasminogen: a new basic variant (PLG B) and population study in Japanese. Vox Sang. 44: 422425.

Nishimukai, H., Kera, Y., Sakata, K., and Yamasawa, K. 1982. Three new variants in the plasminogen system. Hum. Hered. 32: 130-132.

Raum, D., Marcus, D., and Alper, C.A. 1979. Genetic control of human plasminogen (PLGN). Clin. Res. 27: 458A.

Raum, D., Marcus, D., and Alper, C.A. 1980. Genetic polymorphism of human plasminogen. Am. J. Hum. Genet. 32: 681-689.

Remmert, L.F. and Cohen, P.P. 1949. Partial purification and properties of a proteolytic enzyme of human serum. J. Biol. Chem. 181: 431-448.

Robbins, K.C. and Summaria, L. 1976. Plasminogen and plasmin. Methods in Enzymology 45: 257-272.

Sakata, Y. and Aoki, N. 19801 Molecular abnormality of plasminogen. J. Bioi. Chem. 255: 54425447. 